TRANSACTIONS OF THE

AMERICAN MATHEMATICAL SOCIETY

Volume 226, 1977

\title{
POTENTIAL PROCESSES
}

\author{
BY
}

R. V. CHACON

\begin{abstract}
ABSTRACr. The prototype of a potential process is a stochastic process which visits the same points in the same order as a Markov process, but at a rate obtained from a nonanticipating time change. The definition of a potential process may be given intrinsically and most generally without mention of a Markov process, in terms of potential theory. The definition may be given more directly and less generally in terms of potentials which arise from Markov processes, or more directly than this, as suitably time-changed Markov processes. The principal purpose of studying the class of potential processes, which may be shown to include martingales as well as Markov processes themselves, is to give a unified treatment to a wide class of processes which has potential theory at its core. That it is possible to do so suggests that potential rather than martingale results are central to the study of Markov processes. Furthermore, this also suggests that it is not the Markov property itself which makes Markov processes tractable, but rather the potential structure which can be constructed with the assistance of the Markov property. The general theory of potential processes is developed in a forthcoming paper. It will be shown there that a Markov process subject to an ordinary continuous nonanticipating time change is a local potential process. It may be seen, by examining examples, that it is necessary to consider randomized stopping times and randomized nonanticipating time changes in the general case. In the forthcoming paper a more general notion than randomized nonanticipating time changes is used to obtain a characterization of potential processes. It is an open problem whether randomization itself is sufficient in the general case, and whether ordinary nonanticipating time changes are sufficient for continuous parameter martingales and Brownian motion on the line. The emphasis in the present paper will be on developing the theory of discrete parameter martingales as a special case of the general theory.
\end{abstract}

1. Introduction. Let $M$ be a Markov process with stationary transition probabilities and let $\left\{T_{t}^{M}, t \in[0, \infty)\right\}$ be its associated semigroup of transformations on the space of measures on the state space. The potential operator $U_{M}$ is the linear operator on the space of measures $\mu$ given by

$$
U_{M} \mu=\int_{0}^{\infty} T_{t}^{M} \mu d t
$$

It is clear from the definition that the potential operator has the property that

Received by the editors April 14, 1975 and, in revised form, August 20, 1975.

AMS (MOS) subject classifications (1970). Primary 60J45, $31 \mathrm{D} 05$.

Key words and phrases. Potential processes. 
if $\mu_{s}$ is the distribution of the Markov process stopped at $s$, where $s$ is a bounded stopping time, then $U_{M} \mu_{s}$ is monotonically decreasing with increasing $s$, if the potential operator is finite. More generally, $U_{m}\left(\mu_{s(1)}-\mu_{s(2)}\right)>0$, where $s(1)<s(2)$ are two bounded stopping times, for any process. It is this fact, that the potential can be used to keep track of the relative stage of development of the process, even with respect to siopping times, which makes potentials useful.

Brownian motion $B$ on $R^{1}$ is recurrent and so $U_{B} \mu$ is infinite if the measure $\mu$ is positive. If the measure $\mu$ is a signed measure with $\mu\left(R^{i}\right)=0$ and with finite first moment, then $U_{B} \mu$ is not only finite but is absolutely continuous with respect to Lebesgue measure and its Radon-Nikodym derivative is given by

$$
\frac{d U_{B} \mu}{d x}=-\int|x-y| \mu(d y) .
$$

It is convenient to refer to this integral as the potential density of $\mu$ (with respect to Brownian motion $B$ ), and to denote it by $p_{\mu}(x)$. Note that the integral $p_{\mu}(x)$ is finite whenever the measure $\mu$ is finite and has finite first moment, even though it may not always be the Radon-Nikodym derivative of the potential with respect to Brownian motion.

The general program is to indicate in detail how potentials can be used in the study of martingales in the present paper, and then to show how to define potential processes and to develop the general theory in a forthcoming paper. Very roughly, if the potential in question is the potential of a Markov process, then its associated potential processes are related to the potential in the same way that martingales are related to the potential given by the operator $U_{B}$. In the present paper some standard and not so standard martingale results are given potential proofs. The results are intended as samples of the sort of theorems which can be proved for general potential processes, and the proofs given are intended as hints of the proofs needed in the general case. The ordinary martingale results represent the work of many authors, some of whom are mentioned in the bibliography. The papers connecting potential theory and Markov processes are so well-known that no explicit reference is made to them. There are several areas which have not been considered. It is possible to give a particularly simple definition of stochastic integral with respect to martingales using the potential function. The key observation is that potential functions reduce stochastic measures to real measures by looking at the potential at each $x$ in $R$. The same construction can be made to apply to potential processes giving, for example, a definition of stochastic integral with respect to arbitrary Markov processes. It is also possible to interpret the quadratic variation of a martingale in terms of the potential, and it seems likely that some of the inequalities of martingale transforms would 
carry over to the general case. More specific conjectures suggested by these considerations are the following:

(1) Let $M$ be a real-valued Markov process with infinitesimal generator $L$ and let $f(x)$ be a function in the domain of $L$ such that $L f=1$. If $Y$ is a stochastic process such that $f\left(y_{t}\right)-t$ is a martingale and such that it has the same hitting probabilities as $M$, then $Y$ and $M$ are equivalent in the sense of having the same joint distributions.

(2) If two processes have the same hitting distributions, then each is a nonanticipating time change of the other.

2. Properties of the potential in $R^{1}$. Let $\mu$ be a measure defined on the Borel field of $R^{1}$, and call a positive measure having total measure one a unit measure, reserving the name probability measure for the measure of the probability space $(\Omega, \mathcal{F}, P)$. The force of $\mu$ is defined by

where

$$
F_{\mu}(x)=\int q(x-y) \mu(d y)
$$

$$
q(x)= \begin{cases}-1, & x<0 \\ 0, & x=0 \\ 1, & x>0\end{cases}
$$

and the potential of $\mu$ is defined by

It follows that

$$
p_{\mu}(x)=\int-|x-y| \mu(d y) \text {. }
$$

$$
p_{\mu}\left(x_{2}\right)-p_{\mu}\left(x_{1}\right)=-\int_{x_{1}}^{x_{2}} F_{\mu}(s) d s,
$$

so that the potential function is either finite or infinite everywhere, and if finite it is uniformly continuous with modulus of continuity bounded by $\mu\left(R^{1}\right)$ since $\left|F_{\mu}(s)\right|<\mu\left(R^{1}\right)$.

A key property of the potential function is that it is monotone for martingales. This follows from the fact that if $\mu_{0}$ is the unit mass at $x_{0}$ and if $\mu_{1}$ is a unit measure with center of mass at $x_{0}$, then $p_{\mu_{1}}(x)<p_{\mu_{0}}(x)$.

LEMMA 2.1. If $\mu_{1}$ is a unit measure with center of mass at $x_{0}$ and if $\mu_{0}$ is the mass at $x_{0}$, then

$$
p_{\mu_{1}}(x)<-\left|x-x_{0}\right|=p_{\mu_{0}}(x) .
$$

Proof. Assume without loss of generality that $x_{0}=0$. That $-|x|$ is the potential of the unit mass at the origin is trivial. That $p_{\mu_{1}}(x)<-|x|$ follows, for $x>0$, from

$$
p_{\mu}(x)=-\int|x-y| \mu(d y)<-\int(x-y) \mu(d y)=-x,
$$


and for $x<0$ from

$$
\begin{aligned}
p_{\mu}(x) & =-\int|x-y| \mu(d y)=-\int|-x+y| \mu(d y) \\
& <-\int(-x+y) \mu(d y)=x .
\end{aligned}
$$

LEMMA 2.2. If $\mu_{1}$ and $\mu_{2}$ are unit measures with the same center of mass then, if $x>0$,

$$
\left|p_{\mu_{1}}(x)-p_{\mu_{2}}(x)\right|<2\left|\int_{x}^{\infty} y \dot{y}\left(\mu_{1}(d y)+\mu_{2}(d y)\right)\right|,
$$

and if $x<0$,

$$
\left|p_{\mu_{1}}(x)-p_{\mu_{2}}(x)\right|<2\left|\int_{-\infty}^{x} y\left(\mu_{1}(d y)+\mu_{2}(d y)\right)\right|,
$$

so that $\lim _{|x| \rightarrow \infty}\left(p_{\mu_{1}}(x)-p_{\mu_{2}}(x)\right)=0$.

Proof. We will prove the lemma for $x>0$.

$$
\begin{aligned}
p_{\mu_{1}}(x) & -p_{\mu_{2}}(x)=\int-|x-y|\left(\mu_{1}(d y)-\mu_{2}(d y)\right) \\
& =\int((x-y)-|x-y|) \mu_{1}(d y)-\int((x-y)-|x-y|) \mu_{2}(d y),
\end{aligned}
$$

since $\int(x-y) \mu_{1}(d y)=\int(x-y) \mu_{2}(d y)$. Further $(x-y)-|x-y|=0$ if $y$ $<x$ and $(x-y)-|x-y|=2(x-y)$ if $y>x$, so that

$$
p_{\mu_{1}}(x)-p_{\mu_{2}}(x)=2 \int_{x}^{\infty}(x-y)\left(\mu_{1}(d y)-\mu_{2}(d y)\right)
$$

from which the lemma follows since $-y<(x-y)<0$ if $0<x<y$.

LEMMA 2.3. If $\mu_{1}$ and $\mu_{2}$ are unit measures such that $p_{\mu_{1}}(x)>p_{\mu_{2}}(x)$, then $\mu_{1}$ and $\mu_{2}$ have the same center of mass.

Proof. Compare $p_{\mu_{1}}(x)$ and $p_{\mu_{2}}(x)$ with $-\left|x-x_{1}\right|$ and $-\left|x-x_{2}\right|$, where $x_{1}$ is the center of mass of $\mu_{1}$ and $x_{2}$ is the center of mass of $\mu_{2}$ in order to show that $x_{1}=x_{2}$. Lemma 2.2 implies that

$$
\lim _{|x| \rightarrow \infty} p_{\mu_{1}}(x)+\left|x-x_{1}\right|=0, \quad \lim _{|x| \rightarrow \infty} p_{\mu_{2}}(x)+\left|x-x_{2}\right|=0 .
$$

Since $p_{\mu_{1}}(x)-p_{\mu_{2}}(x)>0$, and

$$
\begin{aligned}
\left|x-x_{2}\right|-\left|x-x_{1}\right|= & \left(p_{\mu_{2}}(x)+\left|x-x_{2}\right|\right) \\
& -\left(p_{\mu_{1}}(x)+\left|x-x_{1}\right|\right)+\left(p_{\mu_{1}}(x)-p_{\mu_{2}}(x)\right),
\end{aligned}
$$

the limits imply that

$$
\liminf _{|x| \rightarrow \infty}\left|x-x_{2}\right|-\left|x-x_{1}\right| \geqslant 0 .
$$


This in turn implies that $x_{1}=x_{2}$ since $\left|x-x_{2}\right|-\left|x-x_{1}\right|$ equals $\left(x_{1}-x_{2}\right)$ if $x>\max \left(x_{1}, x_{2}\right)$ and it equals $\left(x_{2}-x_{1}\right)$ if $x<\inf \left(x_{1}, x_{2}\right)$.

A sequence of measures $\left\{\mu_{n}\right\}$ is said to converge weakly to $\mu$, and write $\mu_{n} \Rightarrow \mu$ provided that $\lim _{n \rightarrow \infty} \int f d \mu_{n}=\int f d \mu$, for each continuous function $f$ having compact support.

LEMMA 2.4. If $\left\{\mu_{n}\right\}$ is a sequence of unit measures and if $\mu_{n} \Rightarrow \mu$, then

$$
\limsup _{n \rightarrow \infty} p_{\mu_{n}}(x)<p_{\mu}(x) .
$$

Proof. Let $g(y)$ be a continuous function having compact support and such that $-|x-y|<g(y)<0$. Then

$$
p_{\mu_{n}}(x)=\int-|x-y| \mu_{n}(d y)<\int g(y) \mu_{n}(d y)<0,
$$

so that

$$
\limsup _{n \rightarrow \infty} p_{\mu_{n}}(x)<\int g(y) \mu(d y)<0 .
$$

Letting $g(y) \rightarrow-|x-y|, \lim \sup _{n \rightarrow \infty} p_{\mu_{n}}(x) \leqslant p_{\mu}(x)$.

The next two lemmas were obtained for $R^{n}$ in [2].

LEMMA 2.5. If $\left\{\mu_{n}\right\}$ is a sequence of unit measures such that $\mu_{n} \Rightarrow \mu$ and such that $\lim _{n \rightarrow \infty} p_{\mu_{n}}\left(x_{0}\right)$ exists for some $x_{0} \in R^{1}$, then $\lim _{n \rightarrow \infty} p_{\mu_{n}}(x)$ exists for all $x \in R^{1}$ and there is a constant $C>0$ such that

$$
\lim _{n \rightarrow \infty} P_{\mu_{n}}(x)=p_{\mu}(x)-C .
$$

Furthermore, if there is a unit measure $\nu$ such that $p_{\mu_{n}}(x)>p_{\nu}(x)$ for all $x \in R^{1}$ and all $n$, then $\mu$ is a unit measure and $C=0$.

Proof. Lemma 2.4 implies that $p_{\mu}\left(x_{0}\right)$ is finite. Since

$$
p_{\mu_{n}}(x)-p_{p_{n}}\left(x_{0}\right)=-\int_{x_{0}}^{x} F_{\mu_{n}}(s) d s,
$$

and since it is easy to see that

$$
\lim _{n \rightarrow \infty}-\int_{x_{0}}^{x} F_{\mu_{n}}(s) d s=-\int_{x_{0}}^{x} F_{\mu}(s) d s,
$$

the first part of the lemma follows at once with $C$ a real constant. That $C>0$ follows from Lemma 2.4 .

To see the second part of the lemma, note that if $p_{\mu_{n}}(x)>p_{\nu}(x)$ where the measures are unit measures, then $\lim _{R \rightarrow \infty} \mu_{n}((-R, R))=1$ uniformly in $n$, since otherwise $\inf _{n} p_{\mu_{n}}(x)=-\infty$ for each $x \in R^{1}$, and hence $\mu$ is a unit measure. Since $p_{\mu}(x)-p_{\nu}(x) \geqslant C>0$ and as this implies $p_{\mu}(x)>p_{\nu}(x)$, we may apply Lemma 2.3 to see that $\mu$ and $\nu$ have the same center of mass, and then Lemma 2.2 to obtain that 


$$
\lim _{|x| \rightarrow \infty} p_{\mu}(x)-p_{\nu}(x)=0 .
$$

Since $p_{\mu}(x)-p_{\nu}(x)>C \geqslant 0$, this implies that $C=0$.

LEMMA 2.6. If $\left\{\mu_{n}\right\}$ is a sequence of unit measures such that $\lim _{n \rightarrow \infty} p_{\mu_{n}}(x)$ exists for all $x \in R^{1}$, then $\mu_{n} \Rightarrow \mu$ for some measure $\mu$, and $\mu$ is uniquely determined by the limit $\lim _{n \rightarrow \infty} p_{p_{n}}(x)$.

Proof. It follows easily by integration by parts that each smooth function $f$ having compact support may be written as

$$
f(y)=\frac{1}{2} \int|y-x| f^{\prime \prime}(x) d x,
$$

and hence for such $f$,

$$
\int f(y) \mu_{n}(d y)=\frac{1}{2} \iint|x-y| \mu_{n}(d y) f^{\prime \prime}(x) d x=\frac{1}{2} \int p_{\mu_{n}}(x) f^{\prime \prime}(x) d x .
$$

Since $f^{\prime \prime}(x)$ is bounded and has compact support, and since $p_{\boldsymbol{\mu}_{n}}(x)$ converges uniformly on bounded intervals $\lim _{n \rightarrow \infty} \int f(y) \mu_{n}(d y)$ exists for smooth functions with compact support. This, as is well known, is sufficient to imply the existence of a unique $\mu$ such that $\mu_{n} \Rightarrow \mu$.

3. Optional sampling. If $y$ is a random variable, denote the distribution of $y$ by $\mu(y)$ so that the potential function of $y, p_{y}(x)=p_{\mu(y)}(x)$. Let $\left\{\mathscr{F}_{t}, t \in T\right\}$ be an increasing family of $\sigma$-fields contained in $\mathscr{F}$. We say that $r(\omega), \omega \in \Omega$, is a stopping time of $\left\{\mathscr{F}_{t}, t \in T\right\}$ provided that $r(\omega)$ is a finite $T$-valued random variable such that for each $t \in T,\{\omega: r(\omega)<t\} \in \mathcal{F}_{t}$. To avoid the excessive use of subscripts the notation $r(k, \omega)$ is used instead of $r_{k}(\omega)$, and for simplicity we sometimes write $r(k)$ instead of $r(k, \omega)$.

A sub (super) martingale is a stochastic process $\left\{y_{t}, \mathscr{F}_{t}, t \in T\right\}$ such that for each bounded stopping time $r, y_{r}$ is an $\mathcal{F}_{r}$ measurable random variable and for each pair $r(1)<r(2)$ of bounded stopping times,

$$
\int y_{r(1, \omega)}(\omega) P(d \omega)<(>) \int y_{r}(2, \omega)(\omega) P(d \omega) \text {. }
$$

A martingale is a process which is both a sub and a supermartingale, so that for each martingale $\left\{y_{t}, \mathscr{F}_{t}, t \in T\right\}$ there is a constant $K$ such that for $r$ a bounded stopping time, $\int y_{r}=K$.

Lemma 3.1a. The stochastic process $\left\{y_{1}, \mathscr{F}_{t}, t \in T\right\}$ is a martingale if and only if for each pair $r(1)<r(2)$ of bounded stopping times,

$$
p_{y_{r(1)}}(x)>p_{y_{(2)}}(x) \text {, }
$$

where $p_{\left.y_{r(}\right)}(x)$ is the potential function of $y_{r(i)}, i=1,2$.

Proof. Suppose that $\left\{y_{t}, \mathscr{F}_{t}, t \in T\right\}$ is a martingale. Then for each real $x$, 
$\left\{x-y_{t}, \mathscr{F}_{t}, t \in T\right\}$ is also a martingale and $\left\{\left|x-y_{t}\right|, \bar{F}_{t}, t \in T\right\}$ is a submartingale. Then

$$
-p_{y_{r(1)}}(x)=\int\left|x-y_{r(1)}\right| \leqslant \int\left|x-y_{r(2)}\right|=-p_{y_{r(2)}}(x) .
$$

Next, suppose that $p_{y_{r(1)}}(x) \geqslant p_{y_{r(2)}}(x)$. Since both measures are unit measures, it follows from Lemma 2.3 that they have the same center of mass, and hence $\int y_{r(1)}=\int y_{r(2)}$. Since this is true for each pair of bounded stopping times, it follows that $\left\{y_{t}, \mathscr{F}_{t}, t \in T\right\}$ is a martingale. This concludes the proof of the lemma.

An important notion in the study of martingales is that of a standard stopping time. A stopping time $r$ is a standard stopping time provided that there exists a sequence $\{r(n)\}$ of bounded stopping times such that $\lim _{n \rightarrow \infty} r(n)=r$ with probability one, and such that $\lim _{n \rightarrow \infty} p_{y_{r(n)}}(x)=p_{y_{r}}(x)$.

LEMMA 3.2. Let $\left\{y_{t}, \mathscr{F}_{t}, t \in T\right\}$ be a martingale. If $r$ is a stopping time of the process, then the following conditions are equivalent:

(a) $r$ is a standard stopping time;

(b) if $\bar{r}(n)=\inf (r, n)$ then $\lim _{n \rightarrow \infty} p_{y_{r(n)}}(x)=p_{y_{r}}(x)$;

(c) there exists a sequence $\{r(n)\}$ of standard stopping times such that $\lim _{n \rightarrow \infty} r(n)=r$ with probability one, and such that $\lim _{n \rightarrow \infty} p_{y_{r(n)}}(x)=p_{y_{r}}(x)$;

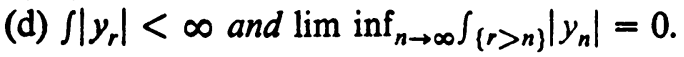

Proof. Since $\left\{y_{t}, \mathscr{F}_{t}, t \in T\right\}$ is a martingale, it follows easily that (a), (b) and (c) are equivalent. To see that (a), (b) and (c) imply (d), note first that it is assumed in (a), (b) and (c) that $\int\left|y_{r}\right|=p_{y_{r}}(0)$ is finite, since $p_{y_{r}}(x)$ is finite. Next, letting $\vec{r}(n)=\inf (r, n)$, note that

$$
\left|y_{\bar{r}(n)}\right|-\left|y_{r}\right|=\chi_{\{r>n\}}\left(\left|y_{n}\right|-\left|y_{r}\right|\right)
$$

where $\chi_{\{r>n\}}$ is the characteristic function of the set $\{r>n\}$. This implies that

$$
p_{y_{r(n)}}(0)-p_{y_{r}}(0)=\int_{\{r>n\}}\left|y_{n}\right|-\int_{\{r>n\}}\left|y_{r}\right| \text {. }
$$

Since $\lim _{n \rightarrow \infty} \int_{\{r>n\}}\left|y_{r}\right|=0$, (b) implies (d). On the other hand, if $\int\left|y_{r}\right|$ is finite it follows from the same equation that $\lim _{\inf _{n \rightarrow \infty}} \int_{\{r>n\}}\left|y_{n}\right|=0$ implies that there exists a subsequence $\{n(k)\}$ such that

$$
\lim _{k \rightarrow \infty} p_{y_{\bar{r}(n(k))}}(0)=p_{y_{r}}(0)
$$

and since $\mu\left(y_{\bar{r}(n(k))}\right) \Rightarrow \mu\left(y_{r}\right)$, that

$$
\lim _{k \rightarrow \infty} p_{y_{\bar{r}(n(k))}}(x)=p_{y_{r}}(x),
$$

by Lemma 2.5 , so that (d) implies (a). Note that since $p_{y_{r(n)}}(x)$ is monotonically 
decreasing, this argument also tells us that there is a further equivalent condition:

(e) $\int\left|y_{r}\right|<\infty$ and $\lim _{n \rightarrow \infty} \int_{\{r>n\}}\left|y_{n}\right|=0$.

Lemma 3.1b. The stochastic process $\left\{y_{t}, \mathscr{F}_{t}, t \in T\right\}$ is a martingale if and only if for each pair $r(1)<r(2)$ of standard stopping times, $p_{y_{r(1)}}(x)>p_{y_{r(2)}}(x)$.

Proof. Follows from Lemma 3.1a, the definition of standard stopping times, and Lemma 3.2(b).

Lemma 3.3. If $\left\{y_{t}, \mathscr{F}_{t}, t \in T\right\}$ is a martingale and $t$ is a standard stopping time, then any stopping time $r<t$ is also a standard stopping time.

PROOF. Since $t$ is a standard stopping time, if $\bar{i}(n)=\inf (t, n)$, then

(i) $p_{y_{(n)}}(x)>p_{y_{i(n+1)}}(x) \geqslant p_{y_{1}}(x)$ and

(ii) $\lim _{n \rightarrow \infty} p_{y_{i(n)}}(x)=p_{y_{t}}(x)$. The sequence $\bar{r}(n)=\inf (n, r)$ satisfies

$$
p_{y_{r(n)}}(x)>p_{y_{r(n+1)}}(x)>p_{y_{(n+1)}}>p_{y_{i}}(x)
$$

by Lemmas 3.1a and 3.1b. Since $\mu\left(y_{\vec{r}(n)}\right) \Rightarrow \mu\left(y_{r}\right)$, Lemma 2.5 implies that $\lim _{n \rightarrow \infty} p_{y_{r(n)}}(x)=p_{y_{r}}(x)$, and hence $r$ is a standard stopping time.

The next result is due to Doob and is known as the optional sampling theorem in the case that $T$ is the set of nonnegative integers.

THeOREM 3.1. Suppose that $\left\{y_{t}, \mathscr{F}_{t}, t \in T\right\}$ is a martingale and suppose that $\{r(t), t \in T\}$ is a family of standard stopping times with $r(t(1))<r(t(2))$ if $t(1)<t(2)$. Then $\left\{y_{r(t)}, \mathscr{F}_{r(t)}, t \in T\right\}$ is a martingale.

Proof. The definition of $\mathscr{F}_{r(t)}$ is the following:

$$
\mathscr{F}_{r(t)}=\left\{A: A \in \mathscr{F}, A \cap\{r(t)<r\} \in \mathscr{F}_{r} \text {, all } r \in T\right\} .
$$

The theorem follows at once from Lemmas 3.3, 3.1a, b.

4. The martingale convergence theorem. There are several proofs of this important theorem. The proof given here is based on the properties of the potential function.

Note first that if $\left\{y_{n}, n=0,1,2, \ldots\right\}$ is a sequence of random variables, and if

$$
y^{*}=\limsup _{n \rightarrow \infty} y_{n}, \quad y_{*}=\liminf _{n \rightarrow \infty} y_{n},
$$

then there exist sequences $\{t(n)\}$ and $\{r(n)\}$ of bounded stopping times such that

$$
y^{*}=\lim _{n \rightarrow \infty} y_{t(n)}, \quad y_{*}=\lim _{n \rightarrow \infty} y_{r(n)} .
$$

This follows easily by considering functions of the form

$$
\sup \left\{y_{n}, s(1) \leqslant n \leqslant s(2)\right\}, \quad \inf \left\{y_{n}, s^{\prime}(1)<n<s^{\prime}(2)\right\},
$$

and then taking the first time after $s(2)$ (or $\left.s^{\prime}(2)\right)$ that the sequence $\left\{y_{n}\right\}$ is 
close to each of these functions. The stopping times $\{t(n)\}$ and $\{r(n)\}$ can be chosen so that $r(1) \leqslant t(1) \leqslant r(2) \leqslant t(2) \leqslant r(3) \leqslant t(3) \leqslant \ldots$, so that if $\left\{y_{n}\right\}$ is a martingale,

$$
p_{y_{r(1)}}(x) \geqslant p_{y_{t(1)}}(x) \geqslant \cdots \geqslant p_{y_{r(n)}}(x) \geqslant p_{y_{t(n)}}(x),
$$

by Lemma 3.1a. If the martingale satisfies the condition that there exists a constant $K$ such that $\int\left|y_{n}\right| \leqslant K$, then $\int\left|y_{t}\right| \leqslant K$ also, for each bounded stopping time. Since $p_{y_{t}}(0)=-\int\left|y_{t}\right|$, the condition implies that

$$
\lim _{n \rightarrow \infty} p_{y_{r(n)}}(x)=\lim _{n \rightarrow \infty} p_{y_{t(n)}}(x)=q(x),
$$

where $q(x)$ is finite. Letting $\mu\left(t_{n}\right)$ be the distribution of $y_{t_{n}}$ and $\mu(t)$ that of $y_{t}$, we have, since $\mu\left(t_{n}\right) \Rightarrow \mu^{*}$ and $\mu\left(r_{n}\right) \Rightarrow \mu_{*}$ where $\mu^{*}$ is the distribution of $y^{*}$ and $\mu_{*}$ is the distribution of $y_{*}$, that $\mu^{*}=\mu_{*}$ by the uniqueness given by Lemma 2.6.

THEOREM 4.1. If $\left\{y_{n}, n=0,1,2, \ldots\right\}$ is a martingale and if there exists a constant $K$ such that $\int\left|y_{n}\right| \leqslant K$, then $\lim _{n \rightarrow \infty} y_{n}$ exists with probability one.

REMARK 4.1. The potential kernel $k(x)=-|x|$ may be regarded as the sum of two kernels $k(x)=k_{1}(x)+k_{2}(x)$, where

$$
k_{1}(x)=\left\{\begin{array}{ll}
x & \text { if } x \leqslant 0, \\
0 & \text { if } x>0,
\end{array} k_{2}(x)= \begin{cases}0 & \text { if } x<0, \\
-x & \text { if } x>0 .\end{cases}\right.
$$

If the potential of a measure $\mu$ is defined using $k_{1}(x)$ and $k_{2}(x)$ as the potential kernels,

and

$$
p_{1 \mu}(x)=\int k_{1}(x-y) \mu(d y), \quad p_{2 \mu}(x)=\int k_{2}(x-y) \mu(d y),
$$

$$
p_{\mu}(x)=p_{1 \mu}(x)+p_{2 \mu}(x) .
$$

These potentials have applications to sub and supermartingales.

5. Uniform integrability. A very important concept in martingale theory is that of uniform integrability. The sequence of random variables $\left\{y_{n}, n>0\right\}$ is defined to be uniformly integrable if

$$
\lim _{N \rightarrow \infty} \int_{\left\{\left|y_{n}\right|>N\right\}}\left|y_{n}(\omega)\right| P(d \omega)=0
$$

uniformly in $n$. A well-known necessary and sufficient condition for uniform integrability is that $\int\left|y_{n}(\omega)\right| P(d \omega)$ be bounded in $n$, and that, if $P(A)=\delta$,

$$
\lim _{\delta \rightarrow 0} \int_{A}\left|y_{n}(\omega)\right| P(d \omega)=0
$$

If $\tau$ is a stopping time of $\left\{y_{n}, n \geqslant 0\right\}$ then $\tau$ satisfies the conditions that $\int\left|y_{\tau}\right|<\infty$ and $\lim _{n \rightarrow \infty} \int_{\{\tau>n\}}\left|y_{n}\right|=0$ if and only if $\left\{y_{\tau \wedge n}\right\}$ is uniformly integrable. This follows from the inequalities 


$$
\begin{aligned}
\int_{A}\left|y_{\tau \wedge n}\right| & \leqslant \int_{A \cap(\tau<n\}}\left|y_{\tau}\right|+\int_{A \cap\{\tau>n\}}\left|y_{n}\right| \\
& <\int_{A}\left|y_{\tau}\right|+\int_{\{\tau>n\}}\left|y_{n}\right|,
\end{aligned}
$$

and gives another characterization of standard stopping times for martingales. In generalizing the notion of a standard stopping time, the characterization in terms of the potential function is the uscful one, however.

The next result gives a necessary and sufficient condition for the uniform integrability of a martingale in terms of its potential functions. It is the following.

LEMMA 5.1. If $\left\{y_{n}, n>0\right\}$ is a martingale then the random variables are uniformly integrable if and only if there exists a unit measure $\mu$ such that $p_{y_{n}}(x)>p_{\mu}(x), x \in R^{l}$.

Proof. It is known that if $\left\{y_{n}, n>0\right\}$ is a uniformly integrable martingale then $\lim _{n \rightarrow \infty} y_{n}=y_{\infty}$ exists almost everywhere and $\left\{y_{n}, n>0, y_{\infty}\right\}$ is a martingale. Lemma 3.1a gives $p_{y_{n}}(x)>p_{y_{\infty}}(x)$. Conversely, if the inequality holds with $p_{\mu}(x)$ replacing $p_{y_{\infty}}(x)$, where $\mu$ is a unit measure, then by the proof of Theorem 4.1 it follows that $\lim _{n \rightarrow \infty} y_{n}=y_{\infty}$ exists almost everywhere and also that $\left\{y_{n}, n>0, y_{\infty}\right\}$ is a martingale with $\int\left|y_{\infty}(\omega)\right| P(d \omega)<\infty$, so that $\left\{y_{n}, n>0\right\}$ is uniformly integrable.

6. Uniqueness. The uniqueness lemma used is the second lemma of this section. The first lemma is a weaker form of it which is given to motivate the ideas.

LEMMA 6.1. Suppose that $\mu_{1}$ and $\mu_{2}$ are two finite measures on $R^{1}$ having finite potential. If $p_{\mu_{1}}(x)=p_{\mu_{2}}(x)$ then $\mu_{1}=\mu_{2}$.

Proof. Let $f$ be a smooth function having compact support. Then

and hence

$$
f(y)=\int-|y-x| f^{\prime \prime}(x) d x,
$$

$$
\begin{aligned}
\int f(y) \mu_{1}(d y) & =\iint-|y-x| f^{\prime \prime}(x) d x \mu_{1}(d y) \\
& =\int f^{\prime \prime}(x) p_{\mu_{1}}(x) d x=\int f^{\prime \prime}(x) p_{\mu_{2}}(x) d x \\
& =\iint-|y-x| f^{\prime \prime}(x) d x \mu_{2}(d y)=\int f(y) \mu_{2}(d y) .
\end{aligned}
$$

Since this relation holds for any smooth function $f$ having compact support, $\mu_{1}=\mu_{2}$.

The next lemma is a stronger version of the first, and in higher dimensions and in a slightly more general form it is known as the domination principle. 
LEMMA 6.2. Let $\mu_{1}$ and $\mu_{2}$ be finite measures on $R^{1}$ having finite potential. If

(a) $p_{\mu_{1}}(x)>p_{\mu_{2}}(x), x \in R^{1}$,

(b) $p_{\mu_{1}}(x)=p_{\mu_{2}}(x)$ on the support of $\mu_{1}$, and

(c) $\lim _{|x| \rightarrow \infty} p_{\mu_{1}}(x)-p_{\mu_{2}}(x)=0$,

then $p_{\mu_{1}}(x)=p_{\mu_{2}}(x), x \in R^{\prime}$ and hence $\mu_{1}=\mu_{2}$.

Proof. Since $p_{\mu_{1}}(x)$ and $p_{\mu_{2}}(x)$ are continuous functions, the set $\{x$ : $\left.p_{\mu_{1}}(x)>p_{\mu_{2}}(x)\right\}$ is an open set which is made up of disjoint open intervals. If one of these intervals is considered $p_{\mu_{1}}(x)=p_{\mu_{2}}(x)$ at its end points and $p_{\mu_{1}}(x)$ is a linear function in the interior, since the interior has $\mu_{1}$ measure zero. Furthermore, since $p_{\mu_{2}}(x)$ is convex, $p_{\mu_{2}}(x)>p_{\mu_{1}}(x)$ and hence $p_{\mu_{2}}(x)=p_{\mu_{1}}(x)$ on the interval. Note that by (c) it is not necessary to distinguish between finite and infinite intervals, and thus the set $\left\{x: p_{\mu_{1}}(x)>p_{\mu_{2}}(x)\right\}$ is empty.

7. Second moments. The results of this section were obtained for $R^{n}$ in [2].

LEMMA 7.1. Let $\mu_{1}$ and $\mu_{2}$ be unit measures having finite potential. If $p_{\mu_{1}}(x)>p_{\mu_{2}}(x), x \in R^{1}$, then

$$
\int\left(p_{\mu_{1}}(x)-p_{\mu_{2}}(x)\right) d x=\int x^{2} \mu_{2}(d x)-\int x^{2} \mu_{1}(d x) .
$$

Proof. Approximate $-x^{2}$ by functions each of which differs from a potential by a constant. This is done by considering the potential of a uniform mass density on a sequence of bounded intervals.

Choose $R>0$ and let $f_{R}(x)$ be given by

$$
f_{R}(x)= \begin{cases}1, & |x|<R \\ 0, & |x|>R\end{cases}
$$

Let $\sigma_{R}$ be the measure with $f_{R}(x)$ for its density, and note that the potential of the measure $\sigma_{R}$ is given by

$$
p_{o_{R}}(x)= \begin{cases}-\left(x^{2}+R^{2}\right), & |x| \leqslant R \\ -2|x| R, & |x|>R .\end{cases}
$$

Then let $v_{R}(x)=p_{\sigma_{R}}(x)+R^{2}$, so that $v_{R}(x)$ is a decreasing function of $R$ for $x$ fixed, and $\lim _{R \rightarrow \infty} v_{R}(x)=x^{2}$.

Note that

$$
\begin{aligned}
\int v_{R}(x) \mu_{2}(d x) & =\int v_{R}(x) \mu_{1}(d x)+\int v_{R}(x) \mu_{2}(d x)-\int v_{R}(x) \mu_{1}(d x) \\
& =\int v_{R}(x) \mu_{1}(d x)+\int p_{o_{R}}(x) \mu_{2}(d x)-\int p_{o_{R}}(x) \mu_{1}(d x) \\
& =\int v_{R}(x) \mu_{1}(d x)+\int\left(p_{\mu_{2}}(x)-p_{\mu_{1}}(x)\right) \sigma_{R}(d x) \\
& =\int v_{R}(x) \mu_{1}(d x)+\int\left(p_{\mu_{2}}(x)-p_{\mu_{1}}(x)\right) f_{R}(x) d x,
\end{aligned}
$$

and letting $R \rightarrow \infty$, the lemma follows. 
LEMMA 7.2. Let $\mu_{1}$ be a unit measure having finite potential and let $\left\{B_{t}\right\}$ be standard brownian motion with initial distribution $\mu_{1}$. If $r$ is any standard stopping time,

$$
\int r(\omega) P(d \omega)=\int x^{2} \mu_{2}(d x)-\int x^{2} \mu_{1}(d x)=\int\left(p_{\mu_{1}}(z)-p_{\mu_{2}}(x)\right) d x,
$$

where $\mu_{2}$ is the distribution of $B_{r}$.

Proof. Since, as is well known, $B_{t}^{2}-t$ is a martingale, the result holds for bounded stopping times. If $\bar{r}(n)=\inf (r, n)$,

$$
B_{\bar{r}(n+1)}-B_{\bar{r}(n)} \perp\left(B_{\bar{r}(n)}-B_{\bar{r}(n-1)}\right)
$$

and the lemma follows by passing to the limit.

8. Balayage of measures. Let $\mu$ be a unit Borel measure and let $I$ be a finite open interval. Define a unit Borel measure $\mu_{I}$ to be the balayage of $\mu$ with respect to $I$, by first defining it for measures which are concentrated on a finite number of points and then passing to the limit. Let $\mu$ be a unit measure concentrated on the points $x_{0}<x_{1}<\cdots<x_{n}$ and suppose that $\mu\left(x_{s}\right)=$ $m_{s}$, where $m_{0}+m_{1}+\cdots+m_{n}=1$. Let $I=(a, b)$ and assume that

$$
x_{0}<\cdots<x_{k}<a<x_{k+1}<\cdots<x_{j-1}<b<x_{j}<\cdots<x_{n} \text {. }
$$

The balayage of $\mu$ with respect to $I$ is the measure $\mu_{I}$ given by

$$
\begin{gathered}
\mu_{I}\left(x_{s}\right)=m_{s}, \quad s=0, \ldots, k, j, \ldots, n, \\
\mu_{I}(a)=\sum_{s=k+1}^{j-1} \frac{m_{s}\left(b-x_{s}\right)}{(b-a)}, \quad \mu_{I}(b)=\sum_{s=k+1}^{j-1} \frac{m_{s}\left(x_{s}-a\right)}{(b-a)} .
\end{gathered}
$$

Note that $\mu_{I}$ agrees with $\mu$ on the complement of closure of $I, \mu_{I}$ is zero on $I$, and $\mu$ gives measure to the end points of $I$ equal to the measure which would be deposited there by Brownian motion (or any other martingale having continuous and unbounded paths) having initial distribution equal to $\mu$ restricted to $I$ (we are ignoring the obvious normalization required since $\mu$ restricted to $I$ is in general not a unit measure). The result of this section compares the potential function of a measure with the potential function of its balayage. It is the following lemma.

LEMMA 8.1. Let $\mu$ be a unit measure having finite potential function, let I be a finite open interval and let $\mu_{I}$ be the balayage of $\mu$ with respect to $I$. Then

(i) $p_{\mu}(x) \geqslant p_{\mu_{l}}(x), x \in R^{1}$,

(ii) $p_{\mu}(x)=p_{\mu_{l}}(x), x \in \bar{I}^{c}$ and

(iii) $p_{\mu_{s}}(x)$ is a linear function for $x \in \bar{I}$.

Proof. The lemma follows easily from the equation

$$
p_{\mu}(x)-p_{\mu}\left(x_{1}\right)=-\int_{x_{1}}^{x} F_{\mu}(s) d s,
$$


where $F_{\mu}$ is the force function of $\mu$, and the corresponding equation for $\mu_{l}$.

9. Conditional potentials and Lebesgue spaces. A measure space $(\Omega, \mathscr{F}, P)$ is a Jebesgue measure space if it is isomorphic to the unit interval plus a countable number of points with discrete measure. It is easy to see that any countably generated measure space is $\sigma$-field isomorphic to a Lebesgue space. In this section it is assumed that the probability space is a Lebesgue space. The point of this assumption is that conditional probabilities will then exist. The language of measure theory is used rather than that of probability.

A partition $\zeta$ is a collection of pairwise disjoint subsets of $\Omega$ whose union is $\Omega$. The elements of $\zeta$ are called partition elements and the partitions may be regarded as the points of a new space which is denoted by $\Omega / \zeta$. The partition elements may be regarded as points of $\Omega / \zeta$ and also as subsets of $\Omega$. Denote by $\mathscr{F}_{\xi}$ those sets of partition elements whose union, when regarded as subsets of $\Omega$, is in $\mathscr{F}$. Given a set $B_{\zeta} \in \mathscr{F}_{\xi}$ define $P_{\xi}\left(B_{\xi}\right)$ by

$$
P_{\zeta}\left(B_{\zeta}\right)=P\left(\bigcup_{S \in B_{\zeta}} S\right)
$$

where $S$ is a partition element. The measure space $\left(\Omega / \zeta, \mathscr{F}_{5}, P_{\zeta}\right)$ is called the factor space of $(\Omega, \mathscr{F}, P)$ with respect to $\zeta$.

Let $\zeta$ be an arbitrary partition of $\Omega$ where $(\Omega, \mathscr{F}, P)$ is a unit Lebesgue measure space, and suppose that each partition element $S$ of $\zeta$ is a measure space itself, that is, suppose that for each $S$ of $\zeta$ there is a $\sigma$-field $\mathscr{F}_{S}$ and a measure $P_{S}$ such that $\left(S, \mathscr{F}_{S}, P_{S}\right)$ is a measure space. Say that $\left(S, \mathscr{F}_{S}, P_{S}\right)$, $S \in \zeta$, is a canonical system of measures provided that

(a) $\left(S, \mathscr{F}_{S}, P_{S}\right)$ is a unit Lebesgue space for almost all $S$ with respect to the factor measure, and

(b) for every set $A \in \mathscr{F}$ the set $A \cap S \in \mathscr{F}_{S}$ for almost all $S$ with respect to the factor measure, $P_{S}(A \cap S)$ is measurable with respect to the factor $\sigma$-field $\mathscr{F}_{5}$, and

$$
P(A)=\int_{\Omega / S} P_{S}(A \cap S) P_{\zeta}(d S) .
$$

An example which makes these ideas clear is the following. Let $(\Omega, \mathscr{F}, \mu)$ be the unit square with the usual Lebesgue sets and Lebesgue measure and let $\zeta$ be the partition having for partition elements the vertical lines. If each vertical line is regarded as a measure space by equipping it with its Lebesgue sets and Lebesgue measure, we have a canonical system of measures with respect to $\zeta$. The factor space $\left(\Omega / \zeta, \mathscr{F}_{\zeta}, P_{\zeta}\right)$ is composed of the vertical lines, the vertical sets, i.e. the sets which are of the form $A \times[0,1]$, and $P_{5}$ is the measure $P$ restricted to vertical sets. This space is isomorphic to $[0,1]$ with Lebesgue sets and Lebesgue measure.

Let $\mathcal{G}$ be an arbitrary collection of subsets of $\Omega$ and denote by $\zeta(\mathcal{G})$ the 
partition generated by $\mathcal{G}$, that is, the partition each of whose elements $S$ is of the form $S=\cap_{A \in S} C(A)$, where $C(A)$ is a set-valued function of $A$, whose value for each $A$ is either $A$ or the complement of $A$. It can be shown that $\zeta(\mathcal{G})=\zeta(F(\mathcal{G}))=\zeta(\mathscr{F}(\mathcal{G})), \mathscr{F}(\mathcal{G})$ is the $\sigma$-field generated by $\mathcal{G}$, and $F^{\prime}(\mathcal{G})$ is the field generated by $\mathcal{G}$. We say that a partition $\zeta$ is measurable if there exists a countable collection $\mathcal{G}$ of $\mathscr{F}$-measurable sets such that $\zeta(\mathcal{G})=\zeta$.

TheOREM 9.1 [12]. Let $(\Omega, F, P)$ be a Lebesgue space. $A$ necessary and sufficient condition for a partition to have a canonical system of measures is that $\zeta$ be measurable, i.e. that $\zeta$ be generated by a countable number of measurable sets.

If $z$ is a random variable and $\mathcal{G}$ a countably generated $\sigma$-field, Theorem 9.1 implies that $E\{z \mid \mathcal{S}\}$ can be written $E\{z \mid S\}$ where $S \in \zeta(\mathcal{G})$ and $E\{z \mid S\}$ is a measurable function of $S$ with respect to the factor $\sigma$-field. Define the conditional potential function of $z$ given $\mathcal{G}$ to be the function of the partition elements $S$ of $\zeta(\mathcal{G})$ given by

$$
p_{z}(x \mid S)=\int-|x-y| P\{z \in d y \mid S\} .
$$

If $B$ is a set of positive probability we define $p_{z}(x \mid B)$ by

$$
p_{z}(x \mid B)=\int-|x-y| P\{z \in d y \mid B\} .
$$

Note that the measures need only be finitely additive for the integrals to be well defined.

Let $z_{0}, z_{1}, \ldots, z_{k}$ be random variables and let $\mathcal{G}$ be the $\sigma$-field which they generate. Each partition element $S$ of the partition $\zeta(\mathcal{G})$ is given by

$$
S=\left\{\omega: z_{0}=x_{0}, \ldots, z_{k}=x_{k}\right\} \text {, }
$$

where $x_{0}, \ldots, x_{k}$ are real numbers. We denote $S$ by $\left(x_{0}, \ldots, x_{k}\right)$. The following lemma follows at once from Theorem 9.1.

LEMMA 9.1. Let $z_{0}, z_{1}, \ldots, z_{k+1}$ be random variables and let $B=\{\omega$ : $\left.\left(z_{0}, \ldots, z_{k-1}\right) \in B_{k}\right\}$ where $B_{k}$ is a $k$-dimensional Borel set. Then

$$
p_{z_{k+1}}(x \mid B)=\int_{B_{k} / 5(8)} p_{z_{k+1}}\left(x \mid\left(x_{0}, \ldots, x_{k}\right)\right) P\left(d x_{0}, \ldots, d x_{k}\right)
$$

where $\mathcal{G}$ is the $\sigma$-field generated by $z_{0}, \ldots, z_{k}$ and where $P\left(d x_{0}, \ldots, d x_{k}\right)$ is another way of writing the factor probability $P_{\zeta}(d S)$.

PRoof. Lemma 9.1 is just a restatement of Theorem 9.1.

LEMMA 9.2. Let $z_{0}, \ldots, z_{k+1}$ be random variables such that

$$
p_{z_{k+1}}\left(x \mid\left(x_{0}, \ldots, x_{k-1}\right)\right)<p_{z_{k}}\left(x \mid\left(x_{0}, \ldots, x_{k-1}\right)\right) \text {, }
$$

for all $x$ for almost all $\left(x_{0}, \ldots, x_{k-1}\right)$ with respect to the factor probability, and such that there is strict inequality for some fixed $\bar{x}$ for $\left(x_{0}, \ldots, x_{k-1}\right) \in B_{k}$, 
where $B_{k}$ has positive factor probability. Then there is an open interval $I, a$ constant $c$ and $a$ set $C_{k} \subset B_{k}$ having positive factor probability, such that

$$
p_{z_{k+1}}\left(x \mid\left(x_{0}, \ldots, x_{k-1}\right)\right)<c<p_{z_{k}}\left(x \mid\left(x_{0}, \ldots, x_{k-1}\right)\right) \text {, }
$$

for $x \in I,\left(x_{0}, \ldots, x_{k-1}\right) \in C_{k}$, and further

$$
P\left\{z_{k} \in I \mid\left(x_{0}, \ldots, x_{k-1}\right)\right\}>0
$$

for $\left(x_{0}, \ldots, x_{k-1}\right) \in C_{k}$.

Proof. A straightforward exercise in measure theory.

10. Embedding theorems. If $\left\{y_{t}, t>0\right\}$ is a martingale and $0=s(0)<s(1)$ $<\ldots$, is a sequence of stopping times, then we say that $\left\{y_{t}, t>0\right\}$ is transformed into $\left\{y_{s(n)}, n>0\right\}$ by $\{s(n), n>0\}$. Two stochastic processes are said to be equivalent if they have the same joint distributions. The first theorem implies that sequences of unrestricted stopping times are too general to be of interest from the point of view of transformations, and the third theorem implies that sequences of standard stopping times are the appropriate sequences to be considered if martingales are to be transformed into martingales.

THEOREM 10.1. Let $\left\{y_{n}, n \geq 0\right\}$ be a discrete parameter stochastic process and let $\left\{B_{t}, t>0\right\}$ be standard Brownian motion. If the distributions of $y_{0}$ and $B_{0}$ are equal, then there exist stopping times $0=s(0)<s(1)<\ldots$, such that $\left\{y_{n}, n>0\right\}$ and $\left\{B_{s(n)}, n \geq 0\right\}$ are equivalent.

Proof. Let $\left\{\bar{y}_{n}, n>0\right\}$ be a stochastic process equivalent to $\left\{y_{n}, n>0\right\}$, and let $\left\{\bar{y}_{n}, n>0\right\}$ be measurable with respect to $\mathscr{F}\left\{B_{t}, 0<t<1\right\}$, the field generated by $\left\{B_{t}, 0<t<1\right\}$. This is possible since $\mathcal{F}\left\{B_{t}, 0<t<1\right\}$ is nonatomic and countably generated and $\mathscr{F}\left\{y_{n}, n>0\right\}$, the field generated by $\left\{y_{n}, n>0\right\}$, is countably generated, so that there is a field isomorphism of $\mathscr{F}\left\{y_{n}, n>0\right\}$ onto a subfield of $\mathscr{F}\left\{B_{l}, 0<t<1\right\}$ which carries $\left\{y_{n}, n>0\right\}$ into an equivalent process $\left\{\bar{y}_{n}, n>0\right\}$. The sequence of stopping times is then defined inductively. Assuming that $0=s(0)<\cdots<s(k)$ have already been defined, let $s(k+1)$ be given by

$$
s(k+1)=\inf \left\{t: B_{t}=\bar{y}_{k+1}, t>1, t>s(k)\right\},
$$

$k=0,1,2, \ldots$ This clearly completes the proof of the theorem.

Theorem 10.2 follows at once from Theorem 10.3 and Lemma 7.2 since all that remains is to show that the stopping times have finite expectations and that their differences are equal to the differences of the second moments of the random variables.

THEOREM 10.2. Let $\left\{y_{n}, n>0\right\}$ be a discrete parameter square integrable martingale and let $\left\{B_{t}, t>0\right\}$ be standard Brownian motion. If the distributions 
of $y_{0}$ and $B_{0}$ are equal, then there exist stopping times having finite expectations $0=s(0)<s(1)<\ldots$, such that the joint distributions of $\left\{y_{n}, n>0\right\}$ and of $\left\{z_{s(n)}\right\}$ are equal, and

$$
\int s(k)-s(k-1)=\int y_{k}^{2}-y_{k-1}^{2} .
$$

Dubins remarks that even if the martingale is not square integrable the stopping times obtained should in some sense be the natural ones. The main theorem of this section addresses itself to this question.

THEOREM 10.3. Let $\left\{y_{n}, n>0\right\}$ be a discrete parameter martingale and let $\left\{z_{t}, t>0\right\}$ be a continuous parameter martingale with continuous and unbounded paths. If the distributions of $y_{0}$ and $z_{0}$ are equal then there exist standard stopping times $0=s(0)<s(1)<\ldots$, such that the joint distributions of $\left\{y_{n}, n>0\right\}$ and of $\left\{z_{s(n)}, n>0\right\}$ are equal.

The first lemma needed in the proof of Theorem 10.3 is the following.

LEMMA 10.1. Let $\left\{z_{t}, t>0\right\}$ be a martingale with continuous parameter and continuous and unbounded sample paths, let $s$ be a standard stopping time and let $I$ be a finite open interval. If $r$ is the stopping time given by

$$
r(\omega)=\inf \left\{t: z_{t} \in I^{c}, t>s(\omega)\right\},
$$

then $r$ is a standard stopping time as well.

Proof. Note that if $\mu(s)$ is the distribution of $z_{s}$ and if $\mu(r)$ is the distribution of $z_{r}$, then $\mu(r)$ is the balayage of $\mu(s)$ with respect to the interval I. Let $A=\left\{\omega: z_{s} \in I\right\}$. Note that $r=s$ on $A^{c}$ so that

$$
r \psi_{A}+r \psi_{A^{c}}=r \psi_{A}+s \psi_{A^{c}}, \quad \bar{r}(n) \psi_{A}+\bar{r}(n) \psi_{A^{c}}=\bar{r}(n) \psi_{A}+\bar{s}(n) \psi_{A^{c}} \text {, }
$$

where $\bar{r}(n)=\inf (r, n)$ and $\bar{s}(n)=\inf (s, n)$. This implies that

$$
\lim _{n \rightarrow \infty} p_{z_{\tilde{F}(n)}}(x)=p_{z_{r}}(x) \text {, }
$$

provided that

$$
\lim _{n \rightarrow \infty} p_{\mu\left(\psi_{1} z_{(n)}\right)}(x)=p_{\mu\left(\psi_{1} z_{r}\right)}(x)
$$

where $\mu\left(\psi_{A} z_{\bar{r}(n)}\right)$ is the distribution of $\psi_{A} z_{\bar{r}(n)}$ and $\mu\left(\psi_{A} z_{r}\right)$ is the distribution of $\psi_{A} z_{r}$. The measure $\mu\left(\psi_{A} z_{r}\right)$ is concentrated at the end points of $I$ and the measure $\mu\left(\psi_{A} z_{\bar{r}(n)}\right)$ is concentrated on the closure of $I$ and it converges weakly to $\mu\left(\psi_{A} z_{r}\right)$, so that the last limit holds. This completes the proof of the lemma.

ProOF OF TheOrem 10.3. The proof is by induction. Assume that standard stopping times $0=s(0)<s(1)<\cdots<s(k)$ are given such that the joint distributions of $\left\{y_{n}, k>n>0\right\}$ and of $\left\{z_{s(n)}, k>n>0\right\}$ are equal, and then find a standard stopping time $s(k+1)$ such that $s(k)<s(k+1)$ and such that the joint distributions of $\left\{y_{n}, k+1 \geqslant n>0\right\}$ and of $\left\{z_{s(n)}, k+1\right.$ $>n>0$ \} are equal. This is done in the following way. Let $R$ be the class of 
standard stopping times such that if $r \in R$,

(i) $r>s(k)$, and

(ii) $p_{z_{r}}\left(x \mid\left(z_{s(0)}, \ldots, z_{s(k)}\right) \in B\right) \geqslant p_{y_{k+1}}\left(x \mid\left(y_{0}, \ldots, y_{k}\right) \in B\right)$,

for all $B$ in the Borel field of $R^{k+1}$ with the property that

$$
P\left(\left(y_{0}, \ldots, y_{k}\right) \in B\right)>0,
$$

where

$$
\begin{aligned}
p_{y_{k+1}}\left(x \mid\left(y_{0}, \ldots, y_{k}\right) \in B\right) \\
\quad=-\int|x-y| P\left(y_{k+1} \in d y \mid\left(y_{0}, \ldots, y_{k}\right) \in B\right) .
\end{aligned}
$$

and

$$
\begin{aligned}
p_{y_{k+1}}\left(x \mid\left(y_{0}, \ldots, y_{k}\right) \in B\right) & \\
& =-\int|x-y| P\left(y_{k+1} \in d y \mid\left(y_{0}, \ldots, y_{k}\right) \in B\right) .
\end{aligned}
$$

Order the standard stopping times in $R$ by almost everywhere inequality and note that every totally ordered subsystem has an upper bound, by Lemma 2.5 . This means that $R$ has a maximal element $r(0)$, by Zorn's lemma. We have that $r(0)>s(k)$ since all the elements of $R$ have this property. Before letting $s(k+1)=r(0)$ we need to check that the joint distributions of $\left\{y_{n}, k+1>\right.$ $n>0\}$ and of $\left\{z_{s(n)}, k+1>n>0\right\}$ are equal. This follows by Lemma 6.1 if it can be shown that

$$
p_{z_{r(0)}}\left(x \mid\left(z_{s(0)}, \ldots, z_{s(k)}\right) \in B\right)=p_{y_{k+1}}\left(x \mid\left(y_{0}, \ldots, y_{k}\right) \in B\right)
$$

for all $B$ with $P\left(\left(y_{0}, \ldots, y_{k}\right) \in B\right)>0$. To see this, note that condition (ii) in the definition of $R$ implies that

$$
\begin{aligned}
p_{z_{r(0)}}\left(x \mid z_{s(0)}\right. & \left.=x_{0}, \ldots, z_{s(k)}=x_{k}\right) \\
& \geqslant p_{y_{k+1}}\left(x \mid y_{0}=x_{0}, \ldots, y_{k}=x_{k}\right)
\end{aligned}
$$

and there is equality, which implies the desired equality (10.1), unless there is a set $B_{0}$, an interval $I$, and a constant $c$ such that

$$
\begin{gathered}
p_{z_{r(0)}}\left(x \mid z_{s(0)}=x_{0}, \ldots, z_{s(k)}=x_{k}\right)>c \\
>p_{y_{k+1}}\left(x \mid y_{0}=x_{0}, \ldots, y_{k}=x_{k}\right)
\end{gathered}
$$

for $x \in I$ and $\left(x_{0}, \ldots, x_{k}\right) \in B_{0}$, where $P\left(\left(y_{0}, \ldots, y_{k}\right) \in B_{0}\right)>0$ and

by Lemma 9.2 .

$$
P\left(z_{r(0)} \in I \mid\left(z_{s(0)}, \ldots, z_{s(k)} \in B_{0}\right)\right)>0,
$$

Next define $r(1)$ by

$$
r(1)=\left\{\begin{array}{rr}
\inf \left\{t: z_{t} \in I^{c}, t \geqslant r(0)\right\} & \text { if } w \in C, \\
\text { where } C=\left\{\omega:\left(z_{s(0)}, \ldots,\right.\right. & \left.\left.z_{s(k)}\right) \in B_{0}\right\}, \\
r, & \text { if } \omega \in C^{c}
\end{array}\right.
$$


and note that $r(1)$ is a standard stopping time and $r(1)>r(0)$ on a set of positive probability, and, by Lemma 8.1 ,

$$
\begin{aligned}
p_{z_{r(1)}}\left(x \mid z_{s}(0)\right. & \left.=x_{0}, \ldots, z_{s}(k)=x_{k}\right) \\
& >p_{y_{k}}\left(x \mid y_{0}=x_{0}, \ldots, y_{k}=x_{k}\right),
\end{aligned}
$$

for all $x, x_{0}, \ldots, x_{k}$ unless equality $(10.1)$ holds. This means that the maximality of $r(0)$ would be contradicted unless (10.1) holds, and this proves the theorem.

11. The interpolation theorems. In this section the following theorem is obtained.

THEOREM 11.1. If $\left\{y_{n}, n \geqslant 0\right\}$ is a discrete parameter martingale then there exists a continuous parameter martingale $\left\{z_{t}, t>0\right\}$ with continuous sample paths such that $\left\{y_{n}, n>0\right\}$ and $\left\{z_{n}, n>0\right\}$ have the same joint distributions.

Note that if the sample paths are not required to be continuous there is nothing to prove since $\left\{z_{t}, t>0\right\}$ could then be defined by setting $z_{t}=y_{n}$, $t \in[n, n-1), n=0,1,2, \ldots$, and it is trivial that $\left\{z_{t}, t>0\right\}$ so defined is a martingale. By using a more complicated construction, a proof similar to that of Theorem 11.1 would yield

THEOREM 11.2. If $\left\{y_{n}, n>0\right\}$ is a discrete parameter martingale such that $P\left\{y_{n} \neq y_{n+1}\right\}=1, n=0,1,2, \ldots$, then there exists a continuous parameter martingale $\left\{z_{t}, t>0\right\}$ with continuous sample paths without intervals of constancy such that $\left\{y_{n}, n>0\right\}$ and $\left\{z_{n}, n>0\right\}$ have the same joint distributions.

Before turning to the proof we introduce some terminology. If $\left\{u_{t}, t>0\right\}$ is a martingale and if $\{r(t), t>0\}$ are standard stopping times with continuous sample paths satisfying for almost all $\omega \in \Omega$,

(i) $r\left(t_{1}, \omega\right)<r\left(t_{2}, \omega\right), t_{1}<t_{2}$, and

(ii) $r(0, \omega)=0$,

then say that $\{r(t), t \geq 0\}$ is a standard time change of $\left\{u_{t}, t>0\right\}$ and call the martingale $\left\{u_{r(t)}, t \geq 0\right\}$ the image of $\left\{u_{t}, t \geq 0\right\}$ under the time change $\{r(t), t>0\}$. Note that if $\left\{u_{t}, t>0\right\}$ has continuous sample paths so does its image, and further, $\left\{u_{t}, t \geqslant 0\right\}$ and its image have the same initial random variable.

LEMMA 11.1. Let $\left\{u_{t}, t>0\right\}$ be a martingale and suppose that $0=s(0)<$ $s(1)<\ldots$, are standard times. Then there exists a standard time change $\{r(t)$, $t>0$ \} such that $r(k)=s(k), k=0,1,2, \ldots$

Proof. Let $\phi^{k}(t), k=1,2,3, \ldots$, be any continuous, one-to-one, and order preserving mapping of $[k-1, k)$ onto $[0, \infty)$ and then let

$$
r(t)=\sup \left[s(k-1), \inf \left(\phi^{k}(t), s(k)\right)\right]
$$


$t \in[k-1, k)$. It is clear that $r(t)$ is a stopping time for each $t$, since stopping times are closed under inf and sup, and it is also clear that the sample paths are continuous. To see that $r(t)$ is a standard stopping time, we have that if $t \in[k-1, k)$, then $r(t)<r(k)=s(k)$ and hence $r(t)$ is a standard stopping time by Lemma 3.3.

Proof OF THeORem 11/1. Apply Theorem 10.3 with the continuous parameter martingale being standard Brownian motion with initial distribution equal to the distribution of $y_{0}$. Denoting it by $\left\{u_{t}, t>0\right\}$ we have standard stopping times $0=s(0)<s(1)<\ldots$, such that the joint distributions of $\left\{u_{s(n)}, n\right\rangle$ $0\}$ and of $\left\{y_{n}, n>0\right\}$ are equal. Applying then Lemma 11.1 we obtain a standard time $\{r(t), t>0\}$ with $r(k)=s(k), k=0,1,2, \ldots$ Then define $z_{t}=u_{r(t)}, t>0$, and the theorem follows at once.

THEOREM 11.3. There exists a stochastic process $\left\{y_{t}, t>0\right\}$ with continuous sample paths and a positive number a such that $\left\{y_{t}, t \in[0, a]\right\}$ and $\left\{y_{t}, t \in[a\right.$, $\infty)\}$ are martingales but $\left\{y_{t}, t \in[0, \infty)\right\}$ is not.

Proof. Let $\left\{B_{t}, t>0\right\}$ be standard Brownian motion starting at the origin, and let $s$ be the standard stopping time obtained by stopping the Brownian motion when it reaches either +1 or -1 . Let $r(1)$ and $r(2)$ be standard stopping times such that $0<r(1)<s<r(2)$. In the rest of the proof it will be convenient to consider $\Omega$ to be the space of paths so that $\omega=\omega(t)$, a continuous real-valued function of $t$ with $\omega(0)=0$ and $\omega(s(\omega))=+1$ or -1 . Let $A$ be a set such that

$$
P\left\{\omega=B_{r(1)} \in A, B_{s}=1\right\}=P\left\{\omega: B_{r(1)} \in A^{c}, B_{s}=1\right\}=\frac{1}{4},
$$

and define the path mapping $T$ for $\omega_{1} \in\left\{\omega: B_{s}=1\right\}$ by $T \omega_{1}=\omega_{2}$, where

$$
\omega_{2}(t)= \begin{cases}\omega_{1}(t), & t<s \\ 1+\left(1-\omega_{1}(t)\right), & t>s\end{cases}
$$

Next, define $\left\{z_{t}, t>0\right\}$ by letting

$$
z_{t}(\omega)= \begin{cases}\omega(t), & \omega \in K_{1} \cup K_{2} \cup K_{3}, \\ T \omega(t), & \omega \in K_{4} \cup K_{5},\end{cases}
$$

where

$$
\begin{aligned}
& K_{1}=\left\{\omega: B_{s}=-1\right\}, \quad K_{2}=\left\{\omega: B_{r(1)} \in A, B_{s}=1, B_{r(2)}>1\right\}, \\
& K_{3}=\left\{\omega: B_{r(1)} \in A^{c}, B_{s}=1, B_{r(2)}<1\right\}, \\
& K_{4}=\left\{\omega: B_{r(1)} \in A, B_{s}=1, B_{r(2)}<1\right\}, \\
& K_{5}=\left\{\omega: B_{r(1)} \in A^{c}, B_{s}=1, B_{r(2)}<1\right\} .
\end{aligned}
$$

Finally, let $y_{t}=z_{\phi_{1}(t)}^{1}, t \in[0,1)$ and $y_{t}=z_{\phi_{2}(t)}^{2}, t \in[1, \infty)$, where $\phi_{1}(t)$ is any one-to-one, continuous and order preserving mapping of $[0,1)$ onto $[0$, $\infty)$ and $\phi_{2}(t)$ is any one-to-one, continuous and order preserving mapping of 
$[1, \infty)$ onto $[0, \infty)$. It follows that $\left\{y_{t}, t \geqslant 0\right\}$ is not a martingale, and that its sample paths are continuous. Lemmas 3.3 and $3.1 \mathrm{~b}$ can be used to see that it is a martingale over $[0,1]$ and $[1, \infty)$. This completes the proof of Theorem 11.3.

\section{BiBLIOGRAPHY}

1. D. G. Austin, G. A. Edgar and A. Ionescu-Tulcea, Pointwise convergence in terms of expectations, Z. Wahrscheinlichkeitstheorie und Verw. Gebiete 30 (1974), 17-26.

2. J. R. Baxter and R. V. Chacon, Potentials of stopped distributions, Illinois J. Math. 18 (1974), 649-656.

3. L. Breiman, On the tail behavior of sums of independent random variables, $Z$. Wahrscheinlichkeitstheorie und Verw. Gebiete 9 (1967), 20-25. MR 37 \#2294.

4. K. E. Dambis, On decomposition of continuous submartingales, Teor. Verojatnost. i Primenen. 10 (1965), 438-448 = Theor. Probability Appl. 10 (1965), 401-410. MR 34 \#2052.

5. J. L. Doob, Stochastic processes, Wiley, New York; Chapman \& Hall, London, 1953. MR $15,445$.

6. L. E. Dubins and G. Schwarz, On continuous martingales, Proc. Nat. Acad. Sci. U.S.A. 53 (1965), 913-916. MR 31 \#2756.

7. L. E. Dubins, On a theorem of Skorohod, Ann. Math. Statist. 39 (1968), 2094-2097. MR 38 \#2837.

8. W. Hall, On the Skorohod embedding theorem, J. Appl. Probability 7 (1970).

9. J. Kiefer, Skorohod embedding of multivariate random variables, and the sample distribution functions, Z. Wahrscheinlichkeitstheorie und Verw. Gebiete 24 (1972), 1-35. MR 49 \#6382.

10. F. Knight, $A$ reduction of continuous square integrable martingales to Brownian motion, Lecture Notes in Math., vol. 190, Springer-Verlag, Berlin and New York, 1970, pp. 19-31.

11. I. Monroe, On embedding right continuous martingales in Brownian motion, Ann. Math. Statist. 43 (1972), 1293-1311. MR 49 \#8096.

12. V. A. Rohlin, On the fundamental ideas of measure theory, Mat. Sb. 25 (67) (1949), 107-150; English transl., Amer. Math. Soc. Transl. (1) 10 (1962), 1-52. MR 11, 18.

13. D. H. Root, The existence of certain stopping times on Brownian motion, Ann. Math. Statist. 40 (1969), 715-718. MR 38 \#6670.

14. S. Sawyer, A remark on the Skorohod representation, $\mathrm{Z}$. Wahrscheinlichkeitstheorie und Verw. Gebiete 23 (1972), 67-74. MR 46 \# 10037.

15. G. Simons, A martingale decomposition theorem, Ann. Math. Statist. 41 (1970), 1102-1104. MR 41 \#6291.

16. H. Kunita and S. Watanabe, On square integrable martingales, Nagoya Math. J. 30 (1967), 209-245. MR 36 \#945.

DEPARTMENT OF MATHEMATICS, UNIVERSITY OF BRITISH COLUMBIA, VANCOUVER, B. C., CANADA 